\title{
Scalable quantum computation in decoherence-free subspaces with trapped ions
}

\author{
Li-Xiang Cen, ${ }^{1,2}$ Z. D. Wang, ${ }^{2,3, *}$ and S. J. Wang ${ }^{1}$ \\ ${ }^{1}$ Department of Physics, Sichuan University, Chengdu 610065, China \\ ${ }^{2}$ Department of Physics \& Center of Theoretical and Computational Physics, University of Hong Kong, \\ Pokfulam Road, Hong Kong, China \\ ${ }^{3}$ National Laboratory of Solid State Microstructures, Nanjing University, Nanjing, China
}

(Received 11 April 2006; published 19 September 2006)

\begin{abstract}
We propose a decoherence-free subspace (DFS) scheme to realize scalable quantum computation with trapped ions. The spin-dependent laser-ion coupling is exploited in the presence of Coulomb interactions. A universal set of unconventional geometric quantum gates is achieved in encoded subspaces that are immune from decoherence by collective dephasing. The scalability of the scheme for an ion-array system is demonstrated, either by adiabatically switching on and off the interactions, or by a fast gate scheme with DFS encoding and noise-decoupling techniques.
\end{abstract}

DOI: 10.1103/PhysRevA.74.032321

PACS number(s): 03.67.Pp, 03.65.Vf, 03.67.Lx

The practical accomplishment of quantum computation requires accurate control of quantum coherent evolution to perform information storage for the quantum bits (qubits), processing of information by quantum gates, and a means of final readout [1]. A quantum computer model based on iontrap systems, which encodes and manipulates information via long-lived internal states of ions, was identified as one promising candidate proposal and has witnessed rapid development in the past decade [2-11]. In recent literature [6,9-11], it was suggested that quantum gates could be realized via certain spin-dependent laser-ion forces in the presence of mutual Coulomb interactions. These theoretical scenarios relax significantly the physical constraints for executing gate operations and hence offer a robust way to implement quantum information processing.

The scalability of the model, i.e., the extension of quantum processors from two qubits to large numbers of ion units, however, is quite challenging due to the growing complexity of the ion vibrational-mode spectrum. An alternative way to achieve scalability, which is pursued by many current efforts, is to devise sophisticated microtrap architecture and design a series of reliable ion shuttling [6,7]. A scaling scenario concerning a large array of ion crystals without ion shuttling has also been suggested in a recent proposal [10], where a prerequisite that the gate interactions should be comparable with the local ion oscillation frequency is indicated.

In this paper we propose a scheme to realize scalable ion-trap quantum computation in decoherence-free subspaces (DFSs) [12] with an extended unconventional geometric scenario [13], which possesses the advantages of the DFS and geometric strategies: the former is immune from decoherence induced by collective dephasing while the latter is thought to be insensitive to certain random errors in the operation process. We exploit the spin-dependent laser-ion coupling in the presence of Coulomb interactions, and then construct a universal set of unconventional geometric quantum gates in encoded subspaces. The potential to scale up the ion-array system for quantum computation without ion shut-

*Electronic address: zwang@hkucc.hku.hk tling is further investigated. Two different interaction configurations, including an adiabatic way of switching on and off the interactions and a scenario to execute rapidly the interaction pulses combined with noise cancellation techniques, are presented.

The system we employed consists of $N$ trapped ions arrayed in a convenient structure. Two hyperfine internal states of each ion, denoted as $|0\rangle$ and $|1\rangle$, are selected to represent physical qubits. In the absence of external forces, the potential $V$ of the system consists of Coulomb interactions between every pair of ions and the external trapping potentials on each ion, which is normally approximated by a secondorder expansion (the harmonic approximation) for small vibrations around the equilibrium configuration $\left(q_{1}^{(0)}, \ldots, q_{N}^{(0)}\right)$. The motional degree of freedom of the ions is therefore treated collectively, and the Hamiltonian in normal coordinates reads

$$
H_{v i b}=\sum_{k} \frac{1}{2 m} P_{k}^{2}+\frac{m}{2} \sum_{k} \omega_{k}^{2} Q_{k}^{2}
$$

Note that the normal coordinates are related to the local one by $Q_{k}=\sum_{j} D_{j k} q_{j}$, where $D$ is an orthogonal matrix that diagonalizes the Hessian $v_{i j}=\left(\partial^{2} V / \partial q_{i} \partial q_{j}\right)\left(q_{1}^{(0)}, \ldots, q_{N}^{(0)}\right)$, and $\omega_{k}(k=1, \ldots, N)$ account for the characteristic frequencies of the normal modes. Suppose that, in order to realize qubitqubit coupling, two of the ions located at $q_{i}$ and $q_{j}$ are acted on by certain acceleration forces that are dependent on the ion internal states, $F_{\mu \alpha}(t)=f_{\mu}(t) \sigma_{\alpha}^{(\mu)}$ with $\mu=i, j$, where $\sigma_{\alpha}^{(\mu)}$ $(\alpha=x, y, z)$ represent the three Pauli operators acting on the states $|0\rangle$ and $|1\rangle$ of the qubit at site $\mu$. The related new interaction term hence takes the form $H_{F}(t)=-q_{i} F_{i \alpha}(t)$ $-q_{j} F_{j \alpha^{\prime}}(t)$. It is readily seen that, by introducing the Fock operators $a_{k}=\left(m \omega_{k} Q_{k}+i P_{k}\right) / \sqrt{2 m \omega_{k}}$ with $\left[a_{k}, a_{k}^{\dagger}\right]=1$, the Hamiltonian in the rotation picture with respect to $H_{v i b}$ takes the following form (setting $\hbar=1$ ):

$$
H(t)=-\sum_{k=1}^{N}\left[g_{i}^{k}(t) \sigma_{\alpha}^{(i)}+g_{j}^{k}(t) \sigma_{\alpha^{\prime}}^{(j)}\right] a_{k} e^{-i \omega_{k} t}+\text { H.c. },
$$

where $g_{\mu}^{k}(t)=\widetilde{D}_{\mu k} f_{\mu}(t)$ for $\mu=i, j$ and $\widetilde{D}_{\mu k}=D_{\mu k} / \sqrt{2 m \omega_{k}}$. 


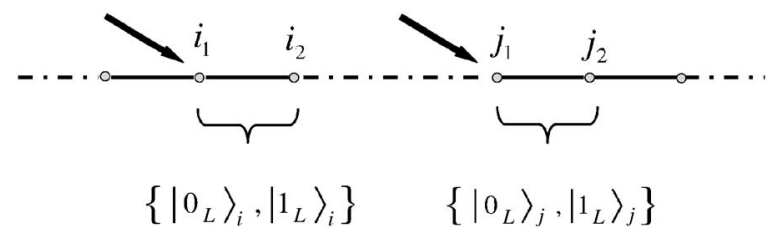

FIG. 1. Schematic of encoded logical qubits for scalable iontrap quantum computation in decoherence-free subspaces.

To investigate the internal state evolution of the ions, we employ a gauged representation with respect to the unitary transformation $G(t)=\exp \left[-i \int_{0}^{t} H(\tau) d \tau\right]$. The evolution operator of the system is given by $U(t)=G(t) U_{g}(t)$, where $U_{g}(t)$ satisfies the covariant equation $i \partial_{t} U_{g}(t)=H^{g}(t) U_{g}(t)$ and the gauged Hamiltonian $H^{g}(t)$ is obtained as [14]

$$
H^{g}(t)=G^{-1} H G-i G^{-1} \partial G / \partial t=\sum_{k=1}^{N} J_{i j}^{k}(t) \sigma_{\alpha}^{(i)} \sigma_{\alpha^{\prime}}^{(j)}+\epsilon_{0}(t),
$$

where

$$
J_{i j}^{k}(t)=\int_{0}^{t}\left[g_{i}^{k}(t) g_{j}^{k}\left(t^{\prime}\right)+g_{i}^{k}\left(t^{\prime}\right) g_{j}^{k}(t)\right] \sin \omega_{k}\left(t^{\prime}-t\right) d t^{\prime},
$$

and $\epsilon_{0}(t)$ is merely a $c$-number parameter. Equation (3) manifests explicitly the "spin-spin" couplings between the ion qubits $i$ and $j$. In particular, as the external force is controlled with a particular configuration such that $\int_{0}^{T} H(t) d t=0$, the transformation $G(T)$ becomes an identity operator and the evolution operator of the system at time $T$ is exactly $U_{g}(T)$

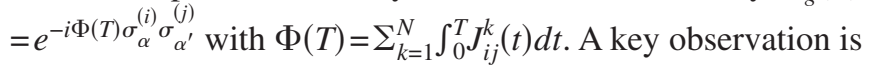
that the generated transformation $U_{g}(T)$ contains only Pauli operators acting on the ion qubits; therefore the induced qubit operation is not affected by the ion motion degree of freedom, and hence is insensitive to the vibrational temperature of the ions. More intriguingly, similar to the case addressed in Ref. [13], the present $U_{g}(T)$ gate may be viewed as an extended version of the unconventional geometric operation [13] whose advantages have been demonstrated in the literature $[4,8,9,15,16]$. This sort of gate will be utilized as a basic one to construct the universal set of gate operations for quantum computation in DFSs.

We employ the pair-bit code by which the logical qubit is encoded in a subspace $C_{i}^{2}$ as

$$
\left|0_{L}\right\rangle_{i}=|0\rangle_{i_{1}} \otimes|1\rangle_{i_{2}}, \quad\left|1_{L}\right\rangle_{i}=|1\rangle_{i_{1}} \otimes|0\rangle_{i_{2}},
$$

where $i=1, \ldots, N / 2$ indexes logical qubits and the schematic array of ions is shown in Fig. 1. Such an encoding constitutes the well-known DFS [12] against the collective dephasing of the system-bath interaction $\Sigma_{i=1}^{N / 2} Z_{i} \otimes B$, where $Z_{i}=\sigma_{z}^{\left(i_{1}\right)}+\sigma_{z}^{\left(i_{2}\right)}$ and $B$ is an arbitrary bath operator. Let us denote $\pi_{x}=\left(\begin{array}{ll}0 & 1 \\ 1 & 0\end{array}\right)$, $\pi_{y}=\left(\begin{array}{cc}0 & -i \\ i & 0\end{array}\right)$ and $\pi_{z}=\left(\begin{array}{cc}1 & 0 \\ 0 & -1\end{array}\right)$ as the three Pauli operators of the encoded logical qubit $\left\{\left|0_{L}\right\rangle,\left|1_{L}\right\rangle\right\}$. A logical controlled-phase flip on two encoded qubits $i$ and $j, e^{i \phi \pi_{z}^{(i)} \pi_{z}^{(j)}}$, could be generated via the aforementioned spin-dependent laser-ion interactions as below. We exploit acceleration forces of the form
$F_{\mu \alpha}(t)=f_{\mu}(t) \sigma_{z}^{(\mu)}$, where the objects could be the two ions $\left\{\mu=i_{1}, j_{1}\right\}$, or alternatively the ions $\left\{\mu^{\prime}=i_{2}, j_{2}\right\}$ with a similar force configuration. According to the previous analysis, as the force configuration is designed so that at time $T$

$$
\eta_{\mu}^{k}(T) \equiv \int_{0}^{T} g_{\mu}^{k}(t) e^{-i \omega_{k} t} d t=0, \quad k=1, \ldots, N,
$$

the specified interactions of Eq. (2) will generate the trans-

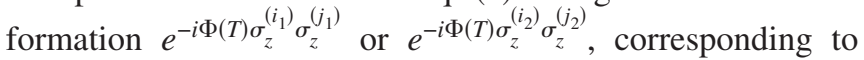
two different addressings of the forces on ion $\mu$ or $\mu^{\prime}$, respectively. Note that the evolution generated by these interactions falls entirely into the encoded subspace $C_{i}^{2} \otimes C_{j}^{2}$ throughout. Moreover, owing to the simple fact that $Z_{i}=Z_{j}$ $=0$, the actions of both the quadratic operators $\sigma_{z}^{\left(i_{1}\right)} \sigma_{z}^{\left(j_{1}\right)}$ and $\sigma_{z}^{\left(i_{2}\right)} \sigma_{z}^{\left(j_{2}\right)}$ in this restricted DFS are equivalent to that of $\pi_{z}^{(i)} \pi_{z}^{(j)}$. Therefore the gate operation $e^{i \phi \pi_{z}^{(i)} \pi_{z}^{(j)}}$ could be exactly achieved by either of the two interacting processes described above.

The commensurability relation (6), which involves all of the ion oscillation modes, might be reached for large- $N$ systems in an adiabatic manner to carry out the pushing forces on the ions. In detail, let us assume that $f_{\mu}(t)$, characterizing the configuration of acceleration forces, is some smooth function of time and satisfies $\left|\dot{f}_{\mu}(t)\right| / \sqrt{2 m \omega_{k}} \ll \omega_{k}$. Moreover, we take $f_{\mu}(t)$ going from $f_{\mu}(0)=0$ and after some finite value reaching an end point $f_{\mu}(T)=0$. It is then direct to see that $\int_{0}^{T_{g}} \mu^{k}(t) e^{-i \omega_{k} t} d t=0$; therefore the relations $\eta_{\mu}^{k}(T)=0$ come into existence for all of the oscillation modes. The generated phase is then obtained as $\Phi(T)=-\Sigma_{k}\left(2 / \omega_{k}\right) \int_{0}^{T} g_{i}^{k}(t) g_{j}^{k}(t) d t$. In addition, the gate time $T$ here is limited by the frequency of the lowest mode (the center-of-mass mode) $\omega_{1}=\omega_{z}$ with $\omega_{z}$ the axial vibrational frequency of the trapping potential. For a trap angular frequency $\omega_{z}=2 \pi \mathrm{MHz}$, the operation period is scaled as $T \gtrsim 10 \mu \mathrm{s}$. Note that the currently proposed DFS scenario to implement qubit operations actually has already tackled partially the intrinsic obstacle associated with the adiabatic process, in which the decoherence is given more time to exert its detrimental effects.

We would also like to give some remarks on the combination of our DFS implementation of gate operations with another scalable approach, i.e., the fast gate scenario by using noise cancellation techniques. In Ref. [10] it was proposed that if the operation speed is comparable with the local ion oscillation frequency, the noise influence due to the complexity of phonon modes could be significantly reduced by designing a multicycle configuration of kicking forces; hence the indicated scalability has been effectively demonstrated. From our formalism, as the commensurability relation (6) is spoiled, the transformation $G_{\mu}(T)$ [or $G_{\mu^{\prime}}(T)$ corresponding to the different ion addressing] contained in the evolution operator indicates actually a noise contribution, say,

$$
G_{\mu}(T)=\exp \left(i \sum_{k} \sum_{\mu=\left\{i_{1}, j_{1}\right\}}\left[\eta_{\mu}^{k}(T) a_{k}+\text { H.c. }\right] \sigma_{z}^{(\mu)}\right) .
$$

It is seen that such an undesirable influence could be suppressed by using two cycles of force pulses with reversed configuration. The reason is that the induced noise effects for 
two-reversed-loop evolution will counteract each other since the coefficients $\eta_{\mu}^{k}(T)$ and $\eta_{\mu^{\prime}}^{k}(T)$ of the noise operators $G_{\mu}(T)$ and $G_{\mu^{\prime}}(T)$ associated with opposite circuits have reversed signs. Note that the present operation scheme actually avoids the requirement of spectroscopic resolution for individual motional sidebands. A challenge now is to control well the periodic laser pulse which should be faster than the frequency of the highest vibrational mode. For a trap containing dozens of to a hundred ions and with a frequency $\omega_{z}=2 \pi \mathrm{MHz}$, an approximate estimation indicates that the vibrational frequencies range from $2 \pi$ to $N \pi \mathrm{MHz}$ [17]. Thus the repetition frequency of the laser beam should be of hundreds of megahertz, which is achievable with the current experimental technique of short laser pulses.

To obtain fully the ability to perform quantum computation, one needs also to construct the general rotation operations for single qubit units. Note that, in the DFS schemes, nontrivial couplings between physical qubits are necessary even to build the gates for single logical qubits. We propose below a similar scheme to realize two universal noncommuting single-qubit gates $\left\{e^{i \phi \pi_{x}}, e^{i \phi^{\prime} \pi_{y}}\right\}$ in the specified DFS by utilizing spin-dependent interactions. In detail, we make use of two different forces $F_{\mu \alpha}(t)$ and $F_{\mu \alpha}^{\prime}(t)(\mu=1,2)$ to derive the two gates $e^{i \phi \pi_{x}}$ and $e^{i \phi \pi_{y}}$, by which $F_{\mu \alpha}(t)=f_{\mu}(t) \sigma_{x}^{(\mu)}$ for the former and $F_{\mu \alpha}^{\prime}(t)=f_{\mu}^{\prime}(t) \sigma_{\alpha}^{(\mu)}$ with $\left\{\sigma_{\alpha}^{(1)}=\sigma_{x}^{(1)}, \sigma_{\alpha}^{(2)}=\sigma_{y}^{(2)}\right\}$ for the latter, respectively. The corresponding interactions of the form (2) should generate the transformations

$$
U_{F}(T)=e^{-i \Phi(T) \sigma_{x}^{(1)} \sigma_{x}^{(2)}}, \quad U_{F^{\prime}}(T)=e^{-i \Phi^{\prime}(T) \sigma_{x}^{(1)} \sigma_{y}^{(2)}}
$$

at time $T$, respectively, provided that the relations (6) with $\mu=1,2$ are satisfied. Note that in the restricted subspace $\left\{\left|0_{L}\right\rangle,\left|1_{L}\right\rangle\right\}$, the actions of the quadratic operators $\sigma_{x}^{(1)} \sigma_{x}^{(2)}$ and $\sigma_{x}^{(1)} \sigma_{y}^{(2)}$ are exactly the same as those of $\pi_{x}$ and $\pi_{y}$. Therefore the transformations of (8) actually offer the two single-qubit gate-operations $e^{i \phi \pi_{x}}$ and $e^{i \phi^{\prime} \pi_{y}}$, respectively.

The protection from state leakage throughout the gating period, however, needs to be scrutinized more carefully. It is recognized that the generated evolution of $U_{F}(t)$ and $U_{F^{\prime}}(t)$ actually employs ion levels out of the DFS; the predicted protection against collective dephasing might be spoiled during the gate operations. For convenience, let us consider a simplified model with only one mode with frequency $\omega$ involved, which accounts physically for sideband addressing by laser beams to select out the particular phonon mode. We assume further a homogeneous dependence of the interactions on the ion internal states, that is, the parameters in (2) satisfy $g_{1}(t)=g_{2}(t)=g(t)$. Note that the evolution of the system now is governed by the overall Hamiltonian

$$
H_{t o t}(t)=H(t)+Z_{i} \otimes B,
$$

where $H(t)$ is assumed to be the interactions associated with $F_{\mu \alpha}(t)$ or $F_{\mu \alpha}^{\prime}(t)$ with specified parameters accordingly. It is readily seen that in the formerly described gauged representation with respect to $G(t)$, one obtains

$$
H_{t o t}^{g}(t)=H^{g}(t)+\widetilde{Z}_{i}(t) \otimes B
$$

where $\tilde{Z}_{i}(t)=G^{-1}(t) Z_{i} G(t)$ and $H^{g}(t)$ has the form of Eq. (3) with a degenerate parameter

$$
J(t)=2 \int_{0}^{t} g(t) g\left(t^{\prime}\right) \sin \omega\left(t^{\prime}-t\right) d t^{\prime} .
$$

In detail, corresponding to the two interactions associated with $F_{\mu \alpha}(t)$ and $F_{\mu \alpha}^{\prime}(t)$, respectively, the form of $\widetilde{Z}_{i}(t)$ can be obtained as

$$
\begin{aligned}
& \widetilde{Z}_{i}(t)=\cos \hat{\eta}^{a}(t) Z_{i}+\sin \hat{\eta}^{a}(t)\left(\sigma_{y}^{(1)}+\sigma_{y}^{(2)}\right), \\
& \widetilde{Z}_{i}^{\prime}(t)=\cos \hat{\eta}^{a}(t) Z_{i}+\sin \hat{\eta}^{a}(t)\left(\sigma_{y}^{(1)}+\sigma_{x}^{(2)}\right),
\end{aligned}
$$

where the operator $\hat{\eta}^{a}(t)=\eta(t) a+\eta^{*}(t) a^{\dagger}$, and $\eta(t)$ $=\int_{0}^{t} g(\tau) e^{-i \omega \tau} d \tau$. It is evident that, even for evolution with perfect parameter controls with $\eta(T)=0$, the occurrence of the final terms in the expression (12) for $\widetilde{Z}_{i}(t)$ and $\widetilde{Z}_{i}^{\prime}(t)$ should inevitably mix the system and bath degrees of freedom and therefore spoil the desired gate operations.

Notably, it happens that the decoherence effects induced above can be reduced effectively via a decoupling process by devising a symmetrized multicircuit evolution. For instance, the first order of decoupling could be achieved via a twocycle refocused performance of the interactions indicated by $\eta\left(t+T_{2} / 2\right)=-\eta(t)$, where $T_{2}$ denotes the whole time period of the two cycles. In view of the relations $\int_{0}^{T_{2}} \eta^{n}(t) d t=0$ with $n$ any odd number and the resulting $\int_{0}^{T_{2}} \sin \hat{\eta}^{a}(t) d t=0$, one obtains readily, by using the Magnus expansion

$$
U_{g}^{t o t}\left(T_{2}\right)=\hat{T} \exp \left(-i \int_{0}^{T_{2}} H_{t o t}^{g}(t) d t\right)=e^{-i\left(h_{1}+h_{2}+\cdots\right) T_{2}},
$$

the first order of the evolution with

$$
\begin{aligned}
h_{1} & =\frac{1}{T_{2}} \int_{0}^{T_{2}} H_{t o t}^{g}(t) d t \\
& =\frac{1}{T_{2}} \int_{0}^{T_{2}} H^{g}(t) d t+\int_{0}^{T_{2}} \cos \hat{\eta}^{a}(t) d t Z_{i} \otimes B .
\end{aligned}
$$

The last term of Eq. (14) actually contributes nothing in our DFS system, therefore the decoherence effects have been effectively removed by the above first-order decoupling process. Physically, for the two cycles with reversed interactions, the ions are pushed in the reverse direction, and the unwanted coupling of the qubits with the vibrational degree of freedom induced by the dissipation has a reverse sign. Due to this sign reversal, the decoherence effects from these two cycles counteract each other. The above decoupling process could be achieved to arbitrarily high orders by iterative application of the multiple refocusing cycles [16].

The expected extension of the above operation scheme with comprehensive DFS encoding and noise-decoupling technique to the scalable system is argued as follows. For a scenario with fast execution of interaction pulses, the refo- 
cusing concept of noise cancellation for the dissipative effects and for decoherence induced by phonon complexity is actually consistent. That is, we have shown that the noise cancellation by the decoupling process is able to remove both kinds of noise provided that the time scale of the interaction pulse is fast enough comparable with the noise frequencies. On the other hand, for the adiabatic pushing scheme, the validity of the extension requires that $\dot{f}(t) \ll \omega_{z}$ and $\dot{f}(t) \gtrsim \tau_{\text {rel }}^{-1}$, where $\omega_{z}$ is the axial vibrational frequency of the trapping potential and $\tau_{r e l}^{-1}$ denotes the relaxation rate of internal states of physical ions.

Before concluding, we would like to note some features of the present geometric DFS scheme for a potential experimental implementation. So far, many experimental investigations of state preparation and state-selective detection of hyperfine states have been reported [18]. The initialization and detection of the DFS encoded registers could be accomplished through laser beam addressing and individually manipulating the ions $i_{2}$. Moreover, our scheme possesses the following advantages. (i) The noise effects of collective dephasing of trapped ions could be tackled [19]. (ii) Since ion shuttling is not necessary in the scheme, one can design the ion array in any convenient geometry. (iii) The scheme is insensitive to the vibrational temperature of the ions. As has been shown, all of the oscillation modes would not spoil the global operation generated by an adiabatic manner of evolution. For the fast gate scenario, by using the noise cancellation technique, there is a very weak influence of the vibrational temperature on the gate fidelity [10].

In summary, we have proposed a DFS scheme to implement scalable ion-trap quantum computation with an extended unconventional geometric approach. Using the spindependent laser-ion interactions, we show that a universal set of quantum gates can be achieved in DFSs, either via adiabatically switching on and off the interactions, or via the scenario to execute rapidly the interaction pulses combined with noise cancellation techniques.

We thank S.-L. Zhu for very helpful discussions. This work was supported by the RGC of Hong Kong (Grant No. HKU7045/05P), the URC fund of HKU, the NSFC (Grant Nos. 10375039, 10429401, and 90503008), and the YSR fund of SCU (Grant No. 200573).
[1] A. Steane, Rep. Prog. Phys. 61, 117 (1998); D. P. DiVincenzo and C. Bennet, Nature (London) 404, 247 (2000).

[2] J. I. Cirac and P. Zoller, Phys. Rev. Lett. 74, 4091 (1995).

[3] C. Monroe et al., Phys. Rev. Lett. 75, 4714 (1995).

[4] A. Sorensen and K. Molmer, Phys. Rev. Lett. 82, 1971 (1999); A. Sorensen and K. Molmer, Phys. Rev. A 62, 022311 (2000); G. J. Milburn, S. Schneider, and D. F. V. James, Fortschr. Phys. 48, 801 (2000).

[5] D. Jonathan, M. B. Plenio, and P. L. Knight, Phys. Rev. A 62, 042307 (2000).

[6] J. I. Cirac and P. Zoller, Nature (London) 404, 579 (2000).

[7] D. Kielpinski, C. Monroe, and D. J. Wineland, Nature (London) 417, 709 (2002).

[8] D. Liebfried et al., Nature (London) 422, 412 (2003).

[9] J.J. Garcia-Ripoll, P. Zoller, and J. I. Cirac, Phys. Rev. Lett. 91, 157901 (2003).

[10] L.-M. Duan, Phys. Rev. Lett. 93, 100502 (2004).

[11] S. L. Zhu, C. Monroe, and L. M. Duan, Europhys. Lett. 73, 485 (2006).
[12] L. M. Duan and G. C. Guo, Phys. Rev. Lett. 79, 1953 (1997); P. Zanardi and M. Rasetti, ibid. 79, 3306 (1997); D. A. Lidar, I. L. Chuang, and K. B. Whaley, ibid. 81, 2594 (1998); L.-A. Wu, P. Zanardi, and D. A. Lidar, ibid. 95, 130501 (2005).

[13] S. L. Zhu and Z. D. Wang, Phys. Rev. Lett. 91, 187902 (2003); S. L. Zhu, Z. D. Wang, and P. Zanardi, ibid. 94, 100502 (2005).

[14] S. J. Wang, F. L. Li, and A. Weiguny, Phys. Lett. A 180, 189 (1993); L.-X. Cen, X. Q. Li, Y. J. Yan, H. Z. Zheng, and S. J. Wang, Phys. Rev. Lett. 90, 147902 (2003).

[15] C. A. Sackett et al., Nature (London) 404, 256 (2000).

[16] L.-X. Cen and P. Zanardi, Phys. Rev. A 71, 060307(R) (2005).

[17] A. M. Steane, Appl. Phys. B: Lasers Opt. 64, 623 (1997); F. Mintert and C. Wunderlich, Phys. Rev. Lett. 87, 257904 (2001).

[18] Ch. Wunderlich and Ch. Balzer et al., Adv. At., Mol., Opt. Phys. 49, 293 (2003);, Ch. Balzer et al., e-print quant-ph/ 0602044.

[19] D. Kielpinski et al., Science 291, 1013 (2001). 